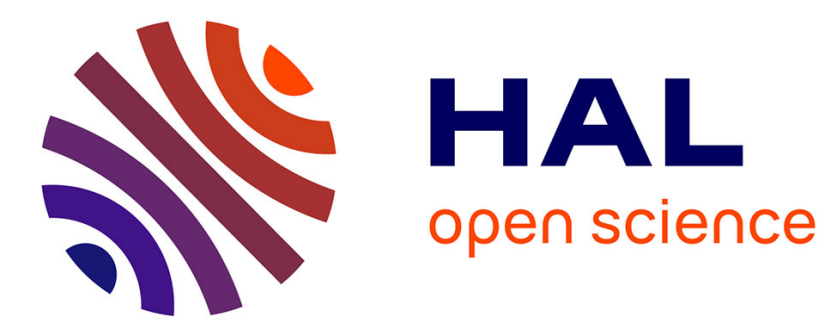

\title{
Photonic nano-structures on (111)-oriented diamond
}

Elke Neu, Patrick Appel, Marc Ganzhorn, Javier Miguel-Sánchez, Margarita Lesik, Vianney Mille, Vincent Jacques, Alexandre Tallaire, Jocelyn Achard, Patrick Maletinsky

\section{- To cite this version:}

Elke Neu, Patrick Appel, Marc Ganzhorn, Javier Miguel-Sánchez, Margarita Lesik, et al.. Photonic nano-structures on (111)-oriented diamond. Applied Physics Letters, 2014, 104 (15), pp.153108. 10.1063/1.4871580 . hal-02493658

\section{HAL Id: hal-02493658 \\ https://hal.science/hal-02493658}

Submitted on 28 May 2021

HAL is a multi-disciplinary open access archive for the deposit and dissemination of scientific research documents, whether they are published or not. The documents may come from teaching and research institutions in France or abroad, or from public or private research centers.
L'archive ouverte pluridisciplinaire HAL, est destinée au dépôt et à la diffusion de documents scientifiques de niveau recherche, publiés ou non, émanant des établissements d'enseignement et de recherche français ou étrangers, des laboratoires publics ou privés.

\section{(c)(1)}

Distributed under a Creative Commons Attribution| 4.0 International License 


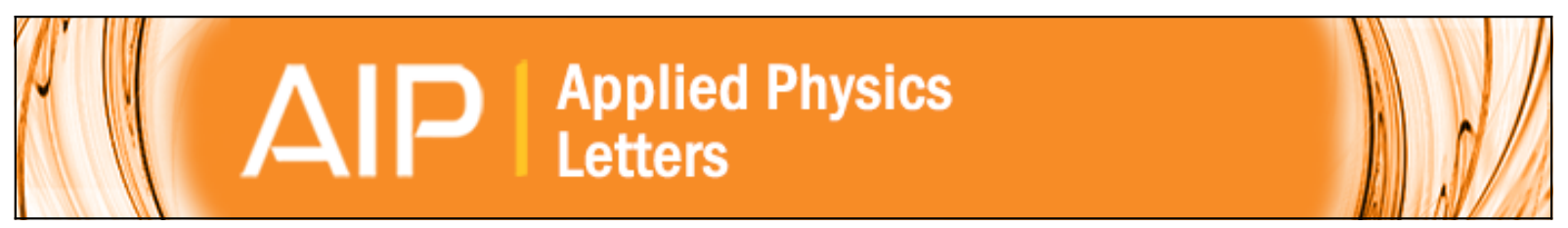

\section{Photonic nano-structures on (111)-oriented diamond}

Elke Neu, Patrick Appel, Marc Ganzhorn, Javier Miguel-Sánchez, Margarita Lesik, Vianney Mille, Vincent Jacques, Alexandre Tallaire, Jocelyn Achard, and Patrick Maletinsky

Citation: Applied Physics Letters 104, 153108 (2014); doi: 10.1063/1.4871580

View online: http://dx.doi.org/10.1063/1.4871580

View Table of Contents: http://scitation.aip.org/content/aip/journal/apl/104/15?ver=pdfcov

Published by the AIP Publishing

\section{Articles you may be interested in}

Native and induced triplet nitrogen-vacancy centers in nano- and micro-diamonds: Half-field electron paramagnetic resonance fingerprint

Appl. Phys. Lett. 104, 063107 (2014); 10.1063/1.4865205

Fabrication of transferrable, fully suspended silicon photonic crystal nanomembranes exhibiting vivid structural color and high-Q guided resonance

J. Vac. Sci. Technol. B 31, 050606 (2013); 10.1116/1.4819297

Nano-structuring in SiGe by oxidation induced anisotropic Ge self-organization

J. Appl. Phys. 113, 104310 (2013); 10.1063/1.4794991

Nano structures via laser interference patterning for guided cell growth of neuronal cells

J. Laser Appl. 24, 042013 (2012); 10.2351/1.4730804

Electrical behavior and oxygen vacancies in BiFeO 3 / [ ( $\mathrm{Bi} 1 / 2 \mathrm{Na} 1 / 2$ ) $0.94 \mathrm{Ba} 0.06$ ] TiO 3 thin film Appl. Phys. Lett. 95, 192901 (2009); 10.1063/1.3259655

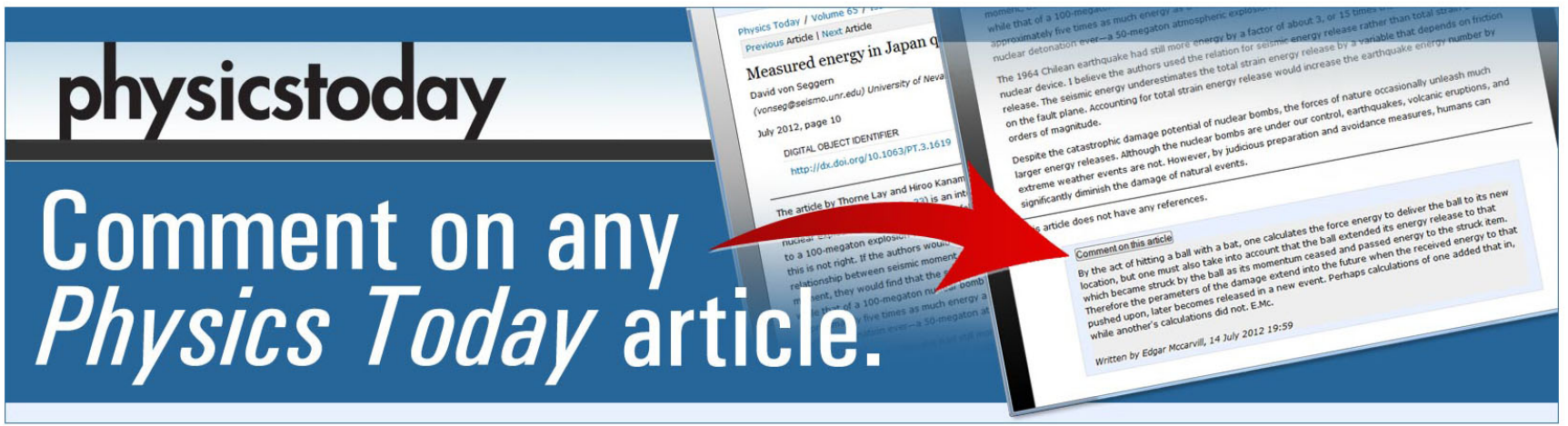




\title{
Photonic nano-structures on (111)-oriented diamond
}

\author{
Elke Neu, ${ }^{1}$ Patrick Appel, ${ }^{1}$ Marc Ganzhorn, ${ }^{1}$ Javier Miguel-Sánchez, ${ }^{1}$ Margarita Lesik, ${ }^{2}$ \\ Vianney Mille, ${ }^{3}$ Vincent Jacques, ${ }^{2}$ Alexandre Tallaire, ${ }^{3}$ Jocelyn Achard, ${ }^{3}$ \\ and Patrick Maletinsky ${ }^{1, a)}$ \\ ${ }^{1}$ Department of Physics, University of Basel, Klingelbergstrasse 82, CH-4056 Basel, Switzerland \\ ${ }^{2}$ Laboratoire Aimé Cotton, CNRS, Université Paris-Sud and Ecole Normale Supérieure de Cachan, \\ F-91405 Orsay, France \\ ${ }^{3}$ Université Paris 13, Sorbonne Paris Cité, Laboratoire des Sciences des Procédés et des Matériaux \\ (CNRS UPR 3407), F-93430 Villetaneuse, France
}

(Received 25 March 2014; accepted 2 April 2014; published online 16 April 2014)

\begin{abstract}
We demonstrate the fabrication of single-crystalline diamond nanopillars on a (111)-oriented chemical vapor deposited diamond substrate. This crystal orientation offers optimal coupling of nitrogen-vacancy (NV) center emission to the nanopillar mode and is thus advantageous over previous approaches. We characterize single native NV centers in these nanopillars and find one of the highest reported saturated fluorescence count rates in single crystalline diamond in excess of $10^{6}$ counts per second. We show that our nano-fabrication procedure conserves the preferential alignment as well as the spin coherence of the NVs in our structures. Our results will enable a new generation of highly sensitive probes for NV magnetometry and pave the way toward photonic crystals with optimal orientation of the NV center's emission dipole. (C) 2014 Author(s). All article content, except where otherwise noted, is licensed under a Creative Commons Attribution 3.0 Unported License. [http://dx.doi.org/10.1063/1.4871580]
\end{abstract}

Diamond nano-structures, including diamond nanopillars for single-photon wave-guiding, ${ }^{1}$ diamond scanning probes for magnetometry, ${ }^{2}$ diamond cantilevers for force-sensing, ${ }^{3}$ or photonic crystals for the efficient cavity coupling of color centers, ${ }^{4,5}$ have been realized recently. A very promising, scalable, and robust approach to engineer diamond-based quantum-devices is top-down nano-fabrication starting from singlecrystalline diamond. ${ }^{6,7}$ Without exception, the above mentioned structures have been fabricated on the (100)-facet of single crystalline diamonds - the most common crystalline orientation and currently the only commercially available type of high-purity diamond material. The functionality of these quantum devices mostly relies on the negatively charged nitrogen-vacancy (NV) defect complex in diamond (NV center). NV centers in diamond align along one of the four equivalent $\langle 111\rangle$ crystal-directions and thus lie at an angle of $54.7^{\circ}$ with respect to $\langle 100\rangle$. This direction forms an important symmetry axis for most of the devices mentioned above, ${ }^{1,2,4,5}$ and the resulting oblique orientation of the NV represents a significant drawback in many cases. For instance, the photon collection efficiency for $\mathrm{NV}$ centers in nanopillars was predicted to be optimal if the emitting dipole is oriented perpendicularly to the pillar's axis; ${ }^{8}$ a situation which is only achieved when the pillar as well as the NV center are aligned along the same $\langle 111\rangle$ axis. Similarly, for scanning NV magnetometry, (111)oriented scanning probes, for which the NV axis can be perpendicular to the sample surface, provide significant improvements compared to existing devices, both in terms of magnetic field sensitivity and ease of interpretation of magnetometry data. ${ }^{9}$ Using nano-cavities in photonic crystals fabricated from single crystalline diamond, the spontaneous emission of color centers can be significantly enhanced. ${ }^{4,5,10}$ Here, the coupling

${ }^{\text {a)} E m a i l: ~ p a t r i c k . m a l e t i n s k y @ u n i b a s . c h ~}$ of a color center to the cavity mode crucially depends on the alignment of the center's dipole moment to the cavity's electric field. ${ }^{4}$ An orientation of this dipole in the plane of the photonic crystal is optimal and is, for the NV center, generally only achievable for (111)-oriented diamond. Despite all these advantages, no (111)-oriented diamond nano-structures have been demonstrated so far. In particular, it is unclear if established diamond nano-fabrication approaches for such structures can be applied to (111)-oriented starting material. Very recently, high-quality, high-purity (111)-oriented chemical vapor deposition (CVD) diamond has become available. ${ }^{11}$ Single NV centers created during CVD growth in this material show very promising properties: ${ }^{12,13}$ almost all NV centers align preferentially along the [111] growth axis and show long spin coherence times $T_{2}>100 \mu$ s. Consequently, it is highly desirable to demonstrate that diamond nano-structures containing single $\mathrm{NV}$ centers can be fabricated in this material.

We here demonstrate the fabrication of nano-photonic structures (nanopillars) from such (111)-oriented, singlecrystalline CVD diamond. We characterize the photonic properties of native NV centers in these nanopillars and measure saturated fluorescence count rates exceeding $10^{6}$ counts per second (cps), which provide evidence for a high collection efficiency for NV centers oriented along the nanopillar axis. Furthermore, we address the photonic properties of the pillars by carrying out finite differential time domain (FDTD) simulations. Importantly, our nano-fabrication procedure conserves the spin properties as well as the preferential alignment of the NV centers, which we demonstrate through electron spin resonance (ESR) and coherent spin manipulation experiments.

Our fabrication recipe is based on previously published procedures for fabricating large, regular arrays of diamond nanopillars. ${ }^{1,8}$ In particular, we are using e-beam lithography $(30 \mathrm{keV})$ in order to pattern cylindrical etch-masks with 
approximately $200 \mathrm{~nm}$ diameter and 550-600 $\mathrm{nm}$ height into a layer of FOX-16 negative electron beam resist (Dow Corning). ${ }^{14}$ A 2 nm titanium layer is deposited onto the diamond prior to resist spinning as an adhesion promoter. Before etching the diamond, the adhesion layer is removed using a short argon sputtering process. The developed mask is transferred into the diamond using inductively coupled plasma reactive ion etching (ICP-RIE, Sentech SI 500) to form the diamond nanopillars. The etch plasma is optimized to create vertical, smooth sidewalls of the pillars as well as a smooth surface in-between the pillars. To this end, we use a plasma containing 50\% argon and oxygen, respectively (gas flow $50 \mathrm{sccm}$ each). The pressure is set to $0.5 \mathrm{~Pa}$, the ICP source is operated at a power of $500 \mathrm{~W}$ together with a bias power of $200 \mathrm{~W}$. The observed etch rate on the (111)oriented diamond was $260 \mathrm{~nm} / \mathrm{min}$. Our pillars were fabricated on the as-grown, cleaned surface of the (111)-oriented single-crystalline CVD diamond investigated in Ref. 12. As demonstrated there, ${ }^{12}$ the NV centers in this sample exhibit near perfect orientation along the growth direction, i.e., out

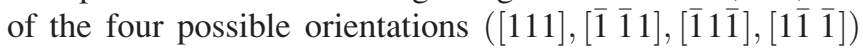
for NV centers, they only occupy the subset along [111] due to the particular dynamics of diamond growth along $\langle 111\rangle$.

Figure 1(a) displays a scanning electron microscope image of typical diamond nanopillars which we fabricate on the (111)-oriented, single-crystalline diamond substrate. Despite the previously reported high density of extended defects in this type of sample, ${ }^{11}$ the etched surface is smooth apart from a low density of triangular etch pits. ${ }^{14}$ The etched pillars show an almost straight shape with a slight asymmetry. The length and diameter of the pillars are approximately $2.3 \mu \mathrm{m}$ and $230 \mathrm{~nm}$, respectively. Additionally and in order to demonstrate the wider applicability of our nanofabrication recipe, we also fabricate nanopillars on a polished, high-purity, polycrystalline diamond sample. Here, we also obtain smooth surfaces with uniformly tall pillars on different grains of the polycrystalline material and only minor preferential etching of grain boundaries. ${ }^{14}$

We characterize the diamond nanopillars using confocal microscopy (NA 0.8, excitation $\mathrm{cw}$ at $532 \mathrm{~nm}$ ). Our setup is equipped with microwave control electronics to perform
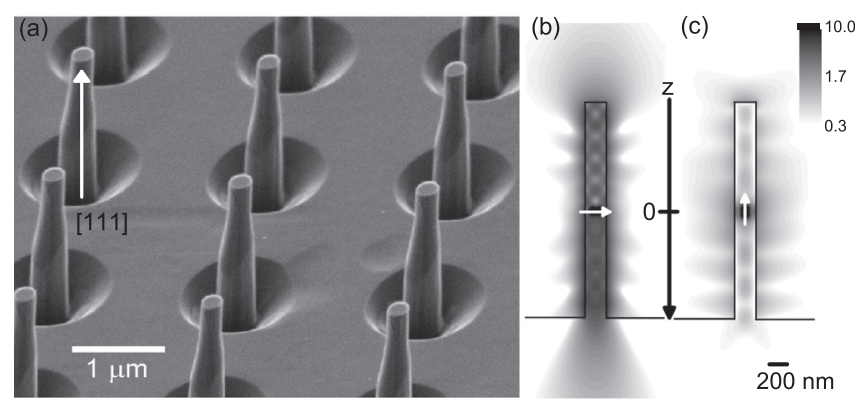

FIG. 1. (a) Scanning electron microscopy image of diamond nanopillars fabricated on a (111)-oriented single-crystalline diamond sample. Note the smooth surfaces in-between the pillars. (b) and (c) FDTD simulations visualizing wave-guiding of the emission from a single dipole oriented perpendicularly to or along the nanopillar axis $(\lambda=700 \mathrm{~nm}$, images show magnitude of E field; white, solid arrows depict dipole orientation). The simulation has been performed for a cylindrical pillar with $230 \mathrm{~nm}$ diameter and $2.3 \mu \mathrm{m}$ length. Note the enhanced coupling efficiency of a dipole oriented perpendicularly to the pillars axis. optically detected ESR experiments on NV centers, ${ }^{15}$ a spectrometer (Acton SP2500, 300 grooves/mm grating) and correlation electronics (Fast ComTec, P7889) to perform second order autocorrelation measurements $\left(\mathrm{g}^{(2)}\right)$ of the fluorescence. For saturation measurements, an avalanche photodiode (Laser Components, Count-250C) with a quantum efficiency of $83 \%$ at $670 \mathrm{~nm}$ is employed.

Figure 2(a) shows a confocal scan of an array of [111]nanopillars. To identify single NV centers, we record the $\mathrm{g}^{(2)}$-function of the fluorescence as displayed in the inset of Fig. 2(b) for a single NV center. Approximately 10\%-20\% of the pillars contain single NV centers as witnessed by a pronounced antibunching dip in the $\mathrm{g}^{(2)}$-function with $\mathrm{g}^{(2)}(0)$ significantly below 0.5 . This probability to find a single NV center in a pillar is consistent with an NV density of 1.5-3 $\mathrm{NVs} / \mu \mathrm{m}^{3}$, which is comparable to the approximate density of native $\mathrm{NV}$ centers of $1 \mathrm{NV} / \mu \mathrm{m}^{3}$ reported in Ref. 12 . The fit (solid line) in the inset of Fig. 2(b) uses the $\mathrm{g}^{(2)}$-function of a three level system, including uncorrelated background. ${ }^{16}$ It is consistent with a signal-to-background ratio (SBR) of approximately 20 and thus confirms very pure single photon emission from our devices. Figure 2(a) also shows that even the pillars that do not contain NV centers exhibit a certain amount of fluorescence, which we assign to broadband background (spectral range $650-800 \mathrm{~nm}$ ) stemming from the starting material. ${ }^{11} \mathrm{We}$ note that this weak background luminescence does not significantly affect the purity of the NV center emission from the nanopillars, which displays the typical fluorescence spectrum of single NV centers including a pronounced zero-phonon-line. ${ }^{14}$

To characterize the photonic properties of our nanopillar devices, we perform saturation-measurements on a subset of pillars, which contain single [111]-oriented NV centers. To separate background emission and fluorescence from single $\mathrm{NV}$ centers, we fit the measured fluorescence intensity $I$ in dependence of the excitation power $P$ using

$$
I(P)=I_{\infty} \frac{P}{P+P_{\mathrm{sat}}}+c_{b} P .
$$

$P_{\text {sat }}$ is the saturation power, $I_{\infty}$ the fully saturated emission rate of the NV center, and the last term $c_{b} P$ accounts for background. ${ }^{17} \mathrm{~A}$ characteristic background corrected saturation curve for a single NV is displayed in Fig. 2(b). Figure 3(a) summarizes $I_{\infty}$ and $P_{\text {sat }}$ obtained for 25 single NV centers. We find an average $I_{\infty}$ of $910 \pm 250 \mathrm{kcps}$ and several single $\mathrm{NVs}$ for which $I_{\infty}$ significantly exceeds 1 Mcps. The saturation power $P_{\text {sat }}$ is below $400 \mu \mathrm{W}$ for the majority of the pillars. Using the saturation curves, we can also estimate the SBR for the fluorescence of single NV centers, which varies significantly between the pillars and can be as high as 60 for low excitation powers. ${ }^{14}$ For an excitation power of $100 \mu \mathrm{W}$, the majority of pillars investigated has an SBR of better than 15 . Consequently, compared to the investigation in bulk, the SBR is enhanced by a factor of 5-10. ${ }^{12}$ Thus, the nanopillars enable very pure single photon emission from NV centers, even in a diamond material exhibiting broad background fluorescence.

In our data in Fig. 3(a), we observe a significant spread of $P_{\text {sat }}$ and $I_{\infty}$, which we assign to different locations of the individual NVs within the nanopillars. Indeed, the NV 

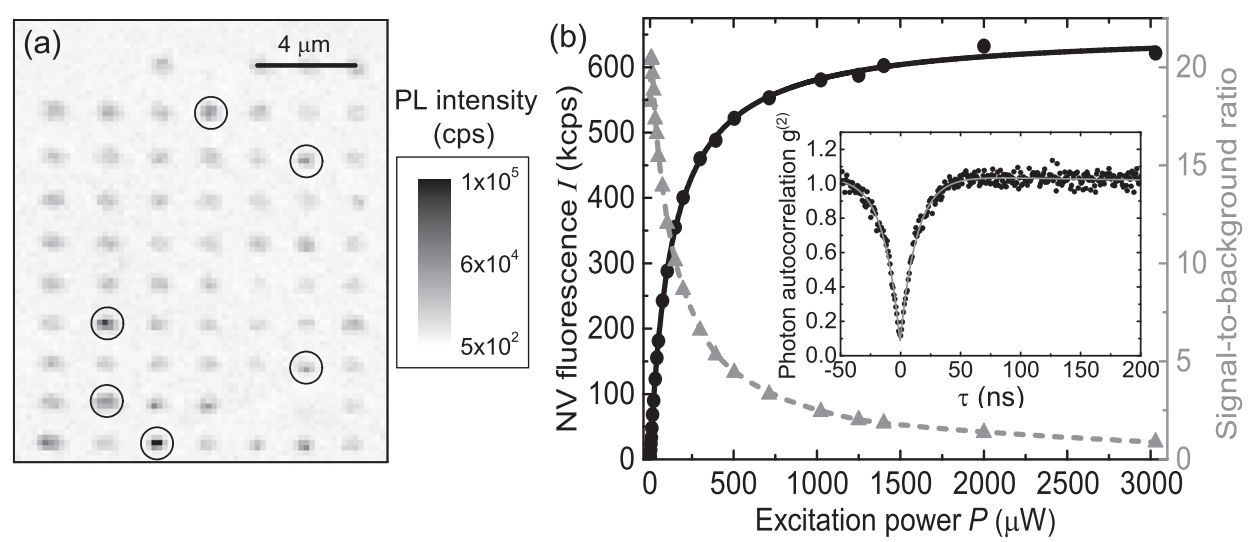

FIG. 2. (a) Confocal fluorescence image of an array of diamond nanopillars. Several pillars with single NV centers $\left(\mathrm{g}^{(2)}(0)=0.2-0.5\right)$ are marked with black, solid circles. The measurement was performed at $120 \mu \mathrm{W}$ of excitation power. (b) SBR and background corrected saturation curve for a single NV center. Inset: $\mathrm{g}^{(2)}$-function of a single NV center. We find $\mathrm{g}^{(2)}(0)=0.12$ without background correction, consistent with a probability of $96 \%$ that the detected photon stems from a single center or a SBR of approximately 20 . The data was recorded at $20 \mu \mathrm{W}$ excitation power.

centers are randomly distributed (radially and axially) in the pillars due to their formation from residual nitrogen during the diamond growth. Excitation of the NV center as well as collection of the fluorescence are both mediated by the pillar's photonic modes [see Figs. 1(b) and 1(c)] and are thus expected to vary depending on the spatial position of the NV center in the pillar. ${ }^{1}$ To investigate the influence of the spatial position, we simulate the electromagnetic fields in the pillar using the FDTD method implemented using commercial software (Lumerical FDTD solutions). First, we simulate the Gaussian excitation field in the nanopillar (cylindrical pillar, diameter $d=230 \mathrm{~nm}, 2.3 \mu \mathrm{m}$ length, refractive index 2.418). From this, we obtain that NV centers close to the pillar's axis experience an excitation field enhanced by $60 \%$ compared to centers close to the pillar's side walls, ${ }^{14}$ thus directly accounting for a spread in the observed $P_{\text {sat. }}$ To estimate the expected spread of $I_{\infty}$, we use a single radiating dipole perpendicular to the pillar axis for the simulation [Fig. 1(b)]. We choose a broadband dipole source with a bandwidth roughly matching the NV center's emission spectrum. From these simulations, we obtain the far field of the pillars. The source is placed in 20 different positions along the nanopillar axis and we extract the collection efficiency $\eta_{\text {coll }}$ (numerical aperture 0.8 ) for 10 different wavelengths $\lambda$ within the NV emission spectrum for each position. As observed in Fig. 3(b), the axial position of the NV strongly influences $\eta_{\text {coll }}$, thus accounting for the observed spread of
$I_{\infty}$. The maximum $\eta_{\text {coll }}$ exceeds the bulk value $(\approx 5 \%)$ by almost an order of magnitude. We note that according to our simulations and previous discussions in Ref. 18, the radial position does not significantly affect $\eta_{\text {coll }}$. For the lifetime of the emitting dipole, however, a placement off axis, in contrast to on axis placement, leads to a suppression of spontaneous emission (maximum simulated suppression: emission rate $\approx 30 \%$ bulk value) due to changes in the local density of states. Taking into account possible non-radiative decays for the NV center, however, it remains questionable if lifetime changes can be observed. As the NV center's emission spans a wavelength range of around $100 \mathrm{~nm}$, it is also instructive to investigate the wavelength dependence of $\eta_{\text {coll }}$. Fig. 3(b) shows a trend for enhanced $\eta_{\text {coll }}$ for longer wavelengths $\lambda$. This is consistent with earlier simulations, ${ }^{18}$ which demonstrated higher transmission of the guided mode through the pillar's top facet for smaller effective diameters $(d / \lambda)$. For comparison and to illustrate the advantages of a [111] oriented NV center, we simulate a dipole parallel to the pillar axis [see Fig. 1(c)]. From our simulations, we conclude that for our collection NA, an NV dipole oriented orthogonally to the wire axis only gives a slight improvement $(\approx 30 \%-40 \%$, depending on position in the wire) in collected fluorescence compared to a dipole parallel to the wire. The high NV fluorescence count rates we observe are thus caused by a combination of this enhancement and improvements of our setup's efficiency compared to previous approaches.

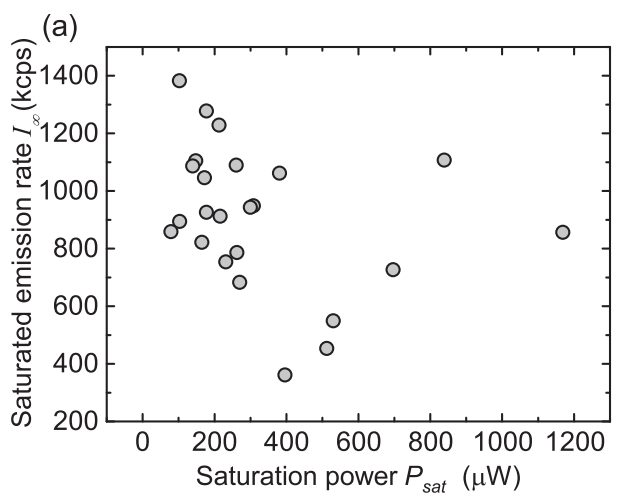

(b)

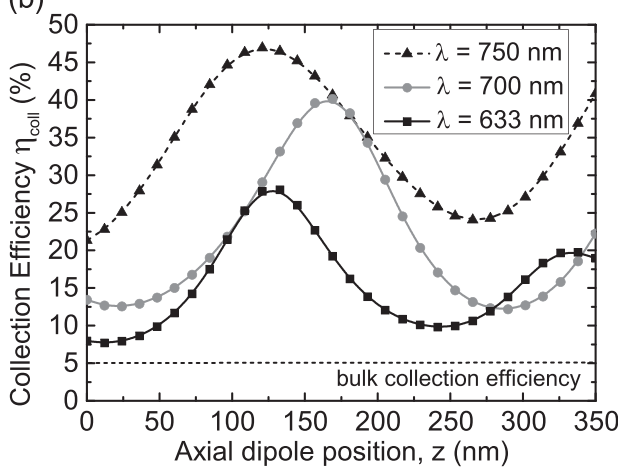

FIG. 3. (a) Saturation power $P_{\text {sat }}$ and maximum saturated emission rates $I_{\infty}$ obtained for 25 single NV centers. (b) Collection efficiency $\eta_{\text {coll }}$ extracted from FDTD simulations for an emitting dipole perpendicular to the nanopillar axis. $\eta_{\text {coll }}$ is shown for three different wavelengths. The emitting dipole is moved along the pillar axis (coordinate $\mathrm{z}$, as defined in Fig. 1) $350 \mathrm{~nm}$ toward the bulk diamond substrate starting from the middle of the pillar. 
(a)

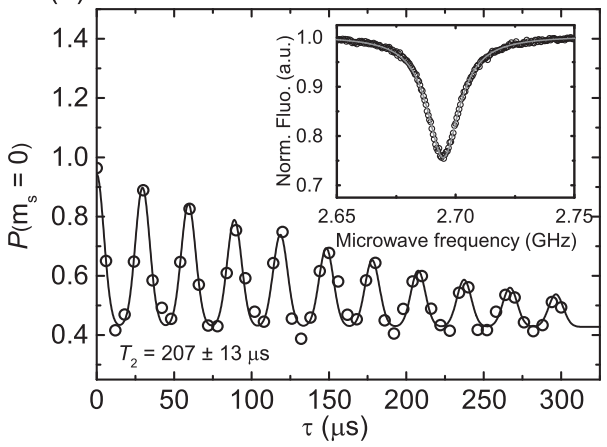

(b)

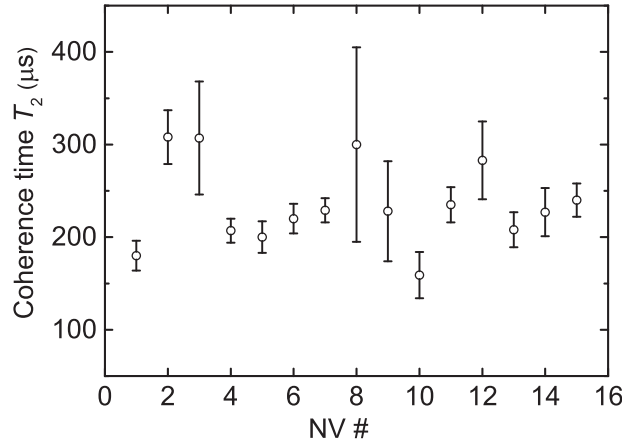

FIG. 4. (a) Spin echo measurement performed on a [111]-oriented NV center in a nanopillar. The solid line is a fit with a sum of Gaussian peaks modulated by a decay envelope. ${ }^{19}$ The inset shows the ESR resonance of a single NV center, displaying a contrast of $25 \%$ for the ESR measurement under continuous laser excitation. (b) Summary of spin coherence times $T_{2}$ for $15 \mathrm{NV}$ centers in nanopillars.
The starting material for the fabrication of the nanopillars has been found to contain NV centers, which orient along the [111] growth axis of the sample with a probability of $97 \% .^{12}$ To verify that the nano-fabrication procedure does not influence the preferential alignment, we apply a static magnetic field aligned with [111]-oriented NV centers. In this configuration, ESR spectra reveal the orientation of the NV centers. From the ESR measurements, we conclude that all 25 single NV centers we investigated are oriented along [111] and the preferential alignment is maintained in the nanopillars. All investigated centers exhibit a very pronounced optically detected ESR resonance, whose contrast under continuous laser excitation reaches $25 \%$. Finally, we verify that our fabrication procedure does not affect spin coherence properties of NV centers in our sample. To that end, we perform Hahn-Echo measurements on a subset of the nanopillars containing single NV centers; the results are summarized in Fig. 4. Before nano-fabrication of the pillars, a $T_{2}$ time of $190 \mu \mathrm{s}$ has been measured for NV centers in the bulk diamond. ${ }^{12}$ For single NVs in the nanopillars, we find $T_{2}$ times between 160 and $310 \mu$ s with an average $T_{2}$ time of $235 \pm 46 \mu \mathrm{s}$.

In summary, we fabricated for the first time nanophotonic structures, namely, nanopillars, with approximately $2 \mu \mathrm{m}$ length and $200 \mathrm{~nm}$ diameter, on high-purity, (111)-oriented single-crystalline CVD diamond. We characterize single native NV centers in these nanopillars and find high saturated fluorescence count rates around $10^{6} \mathrm{cps}$. The nano-fabrication procedure conserves the preferential alignment of the centers as well as the spin coherence properties. This demonstration of nano-device fabrication on (111)-oriented diamond paves the way toward diamond scanning probes for magnetometry and photonic crystals with optimal orientation of the NV center's emission dipole. Ultimately, isotopically pure diamond growth in combination with the formation of NV centers in a nanometer thin layer ( $\delta$-doping) can lead to devices with a controlled depth of the NV centers as well as ultra-long spin coherence times. ${ }^{20,21}$

We gratefully acknowledge financial support through the NCCR QSIT, a competence center funded by the Swiss NSF, and through SNF Grant No. 200021_143697/1. This research has been partially funded by the European
Commission's 7. Framework Program (FP7/2007-2013) under Grant Agreement No. 611143 (DIADEMS).

${ }^{1}$ T. Babinec, B. Hausmann, M. Khan, Y. Zhang, J. Maze, P. Hemmer, and M. Loncar, Nat. Nanotechnol. 5, 195 (2010).

${ }^{2}$ P. Maletinsky, S. Hong, M. Grinolds, B. Hausmann, M. Lukin, R. Walsworth, M. Loncar, and A. Yacoby, Nat. Nanotechnol. 7, 320 (2012).

${ }^{3}$ P. Ovartchaiyapong, L. M. A. Pascal, B. A. Myers, P. Lauria, and A. C. B. Jayich, Appl. Phys. Lett. 101, 163505 (2012).

${ }^{4}$ A. Faraon, C. Santori, Z. Huang, V. M. Acosta, and R. G. Beausoleil, Phys. Rev. Lett. 109, 033604 (2012).

${ }^{5}$ J. Riedrich-Möller, L. Kipfstuhl, C. Hepp, E. Neu, C. Pauly, F. Mücklich, A. Baur, M. M. Wandt, S. Wolff, M. Fischer, S. Gsell, M. Schreck, and C. Becher, Nat. Nanotechnol. 7, 69 (2012).

${ }^{6}$ I. Aharonovich, A. D. Greentree, and S. Prawer, Nat. Photonics 5, 397 (2011).

${ }^{7}$ M. Loncar and A. Faraon, MRS Bull. 38, 144-148 (2013).

${ }^{8}$ B. J. Hausmann, M. Khan, Y. Zhang, T. M. Babinec, K. Martinick, M. McCutcheon, P. R. Hemmer, and M. Loncar, Diamond Relat. Mater. 19, 621 (2010).

${ }^{9}$ L. Rondin, J. Tetienne, S. Rohart, A. Thiaville, T. Hingant, P. Spinicelli, J.-F. Roch, and V. Jacques, Nat. Commun. 4, 2279 (2013).

${ }^{10}$ B. J. M. Hausmann, B. Shields, Q. Quan, P. Maletinsky, M. McCutcheon, J. T. Choy, T. M. Babinec, A. Kubanek, A. Yacoby, M. D. Lukin, and M. Loncar, Nano Lett. 12, 1578 (2012).

${ }^{11}$ A. Tallaire, J. Achard, A. Boussadi, O. Brinza, A. Gicquel, I. Kupriyanov, Y. Palyanov, G. Sakr, and J. Barjon, Diamond Relat. Mater. 41, 34 (2014).

${ }^{12}$ M. Lesik, J.-P. Tetienne, A. Tallaire, J. Achard, V. Mille, A. Gicquel, J.-F. Roch, and V. Jacques, Appl. Phys. Lett. 104, 113107 (2014).

${ }^{13}$ J. Michl, T. Teraji, S. Zaiser, I. Jakobi, G. Waldherr, F. Dolde, P. Neumann, M. W. Doherty, N. B. Manson, J. Isoya, and J. Wrachtrup, Appl. Phys. Lett. 104, 102407 (2014).

${ }^{14}$ See supplementary material at http://dx.doi.org/10.1063/1.4871580 for further technical details on sample growth and nanofabrication procedure as well as additional data on FDTD simulations and spectral and photonic properties of NVs.

${ }^{15}$ A. Gruber, A. Dräbenstedt, C. Tietz, L. Fleury, J. Wrachtrup, and C. von Borczyskowski, Science 276, 2012 (1997).

${ }^{16}$ R. Brouri, A. Beveratos, J. Poizat, and P. Grangier, Opt. Lett. 25, 1294 (2000).

${ }^{17}$ C. Kurtsiefer, S. Mayer, P. Zarda, and H. Weinfurter, Phys. Rev. Lett. 85, 290 (2000).

${ }^{18}$ I. Friedler, C. Sauvan, J. P. Hugonin, P. Lalanne, J. Claudon, and J. M. Gérard, Opt. Express 17, 2095 (2009).

${ }^{19}$ L. Childress, M. Dutt, J. Taylor, A. Zibrov, F. Jelezko, J. Wrachtrup, P. Hemmer, and M. Lukin, Science 314, 281 (2006).

${ }^{20}$ G. Balasubramanian, P. Neumann, D. Twitchen, M. Markham, R. Kolesov, N. Mizuochi, J. Isoya, J. Achard, J. Beck, J. Tissler, V. Jacques, P. Hemmer, F. Jelezko, and J. Wrachtrup, Nat. Mater. 8, 383 (2009).

${ }^{21}$ K. Ohno, F. J. Heremans, L. C. Bassett, B. A. Myers, D. M. Toyli, A. C. B. Jayich, C. J. Palmstrom, and D. D. Awschalom, Appl. Phys. Lett. 101, 082413 (2012). 